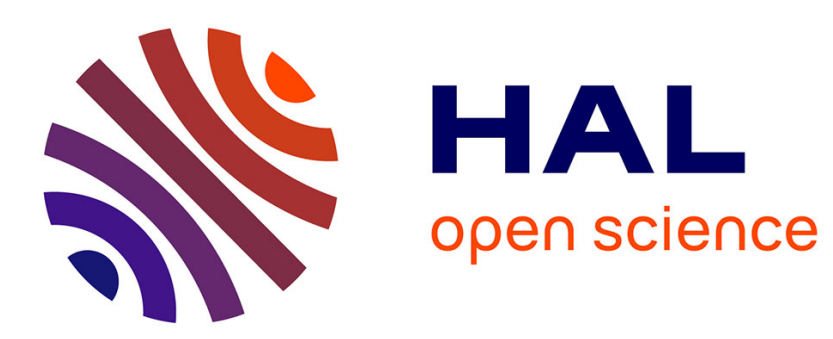

\title{
Some thoughts on multipolar sources in a moving medium and the structure of the wave equation
}

\author{
R. Musafir
}

\section{To cite this version:}

R. Musafir. Some thoughts on multipolar sources in a moving medium and the structure of the wave equation. Journal de Physique IV Proceedings, 1994, 04 (C5), pp.C5-943-C5-946. 10.1051/jp4:19945206 . jpa-00252890

\section{HAL Id: jpa-00252890 https://hal.science/jpa-00252890}

Submitted on 1 Jan 1994

HAL is a multi-disciplinary open access archive for the deposit and dissemination of scientific research documents, whether they are published or not. The documents may come from teaching and research institutions in France or abroad, or from public or private research centers.
L'archive ouverte pluridisciplinaire HAL, est destinée au dépôt et à la diffusion de documents scientifiques de niveau recherche, publiés ou non, émanant des établissements d'enseignement et de recherche français ou étrangers, des laboratoires publics ou privés. 


\title{
Some thoughts on multipolar sources in a moving medium and the structure of the wave equation
}

\author{
R.E. MUSAFIR
}

Acoust. \& Vibr. Lab., Dept. Mech. Eng., COPPE and Hydraulics Dept., EE Universidade Federal do Rio de Janeiro, CP. 68503, 21945-900 Rio de Janeiro, Brazil

\begin{abstract}
The procedure of derivation of the wave equation is reviewed, it been pointed out that, while for a medium at rest there is a clear correspondence between point multipole sources originating from the expansion of a general source term and with multipoles resulting from singularities in the continuity and momentum equations, this is not so for a moving medium. Thus, a classification of source multipole order based on the expansion of a general source may lack physical significance. Starting from 'natural' monopole and dipole operators, identified in the source function, different families of multipole operators are defined, based on the expansion of the appropriate terms. It is shown that the wave operator has a structure similar to that of the source function, being composed of particular multipole operators. Applications of these features in analyzing different formulations for the aerodynamical noise problem and in optimizing the choice of equivalent source terms are highlighted.
\end{abstract}

\section{INTRODUCTION}

In a medium at rest, the characterization of the multipole order of a given source term for the wave equation is straightforward, being based on the number of space derivatives present in that term: a $2^{n}-$ pole is associated with the $n^{\text {th }}$ divergence of a tensor field of order $n$. Thus, a monopole $(n=0)$ contains no space derivative, a dipole $(n=1)$ has a single $\nabla$. operator, and so on. In this case, any mathematical source distribution can easily be given (at least to some extent) a physical meaning, since a point multipole of given order can always be interpreted as having being generated by a cancelling pair of pont sources of imediately inferior order, while monopole and dipole sources can be associated with sources in the fundamental equations. In a moving medium, this question becomes more elaborate, there existing natural forms of presenting the source terms if they are expected to retain any physical meaning. This paper addresses this point, discussing also implications of these ideas in understanding the structure of the wave operator.

\section{NON-HOMOGENOUS WAVE EQUATION}

\section{1 - Derivation}

The equation describing the linear propagation of sound waves in a continuous source free medium is obtained, for the situations where such an equation exists, by the combination of the continuity and momentum equations, henceforth called respectively I and $\boldsymbol{I}$ (bold face notation is used for II to remind that it represents a vector equation), which envolve, as variables, pressure, density and velocity $(p, \rho, v)$. Also needed is the energy equation or a constitutive relation between pressure and density fluctuations, which introduces the square sound speed, $c^{2}$. 
In order to eliminate fluctuations of $\mathbf{v}$ (or of $\rho \mathbf{v}$ ), linear diferential operators, $\mathscr{M}$ and $\mathscr{D} .-\mathscr{D}$ being a vector operator - are applied to the linearized forms of $I$ and $\Pi, I_{0}$ and $\Pi_{0}$, an equation for a single variable, say $\pi$, characterized by the wave operator $\mathscr{L}$, being obtained as

$$
\mathscr{L}[\pi]=\mathscr{M}\left[\mathrm{I}_{0}\right]+\mathscr{D} \cdot\left[\Pi_{0}\right]=0
$$

If we consider now the existence of a source term $\mathrm{Q}$ in (1), it is expected that it can be traced back to the fundamental equations. So, in order to have a better insight on the meaning of this term, it is convenient to work with the non-homogenous forms of $I_{0}$ and $\boldsymbol{\Pi}_{0}$, which include right hand sides $q$ and $\mathbf{f}$, respectively, representing sources of mass (more properly volume) and momentum per unit mass or volume. These terms can be associated with real external sources, with the effect of boundaries or with parts of the density and velocity fields that are more properly related to sound generation than to its propagation. The non-homogenous wave equation thus obtained is

$$
\mathscr{L}[\pi]=\mathrm{Q}=\mathscr{M}[\mathrm{q}]+\mathscr{D} \cdot[\mathrm{f}]
$$

Equation (2) shows that there is a natural way of writing source terms, which will be different, according to the operations, represented by $\mathscr{M}$ and $\mathscr{D}$., to be performed on the fundamental equations. These operations, of course, depend on the mean properties of the situation under discussion.

\section{2 - Source Terms}

We start by examining the simplest case, that of an homogenous medium at rest. Then, $\mathscr{L}$ is the $D^{\prime}$ Alembert operator and $\mathscr{M}$ and $\mathscr{D}$ are given by $\partial / \partial \mathrm{t}$ and $-\nabla$, the equation corresponding to (2) being written, for $\pi$ taken as pressure fluctuations p' as

$$
\left(\frac{1}{c_{0}{ }^{2}} \frac{\partial^{2}}{\partial^{2}}-\nabla^{2}\right) p^{\prime}=Q=\frac{\partial q}{\partial t}-\nabla . f
$$

where $c_{0} 2$ is the linearized value of $c^{2}$.

It is well known that a scalar source function ( $Q, q, \partial q / \partial t, \nabla . f)$ corresponds to a monopole source distribution, and also that a general $Q$ can be written, through the Taylor expansion of the operator's Green's function about a fixed source point, as a sum of point multipoles of ascending order at that particular point (e.g. [1]). In the context of equation (3), a point dipole is both the second term of the expansion for a general $Q$ and the first term in the corresponding expansion for the $\mathrm{f}$ term, which can be called a dipole source distribution. The field of the monopole distribution $\partial \mathrm{q} / \partial t=-\nabla . f$ and that of the dipole distribution $\mathrm{f}$ are, of course, identical.

When there is movement, as $\mathscr{D}$ is not necessarily given by $-\nabla$ and $\mathscr{M}$ may also depend on the space variable (the most likely candidate being the linearized material time derivative $D_{0} / \mathrm{Dt}$ ), there will be no simple relationship between source terms originating from the continuity and from the momentum equations. It follows then that the appropriate source function to be expanded is not $Q$, but its fundamental constituents, $q$ and $f$. Thus, two different families of multipoles will arise: one stemming from the volume velocity monopole distribution $\mathrm{q}-\mathrm{i}$.e., from sources in the continuity equation - and the other from the volume aceleration dipole distribution $f$ corresponding to sources in the momentum equation. As $q$ and $f$ are 'natural' source terms, one may call the operators $\mathscr{M}$ and $\mathscr{D}$. by volume velocity monopole and volume acceleration dipole, respectively.

Higher order source distributions can be easily generated from $q$ and $f$, the corresponding multipolar operators being defined from $\mathscr{M}$ and $\mathscr{D}$., through the addition, for each unit increase in order, of $-\nabla$. on the right side of the operators. A volume acceleration quadrupole operator is given by $-\mathscr{D}$. ( $\nabla$.$) while the volume velocity quadrupole operator is \mathscr{M}(\nabla . \nabla$.$) .$

A third type of source may also be called natural, being equally important and giving rise to another family of sources: the volume displacement monopole distribution $\mathrm{q}^{*}$, which relates to $\mathrm{q}$ by $\mathrm{q}=\mathrm{D}_{0} \mathrm{q}^{*} / \mathrm{Dt}$. Thus, $\mathscr{H}\left(\mathrm{D}_{0} / \mathrm{Dt}\right)$ is the volume displacement monopole operator while the corresponding quadrupole operator, for instance, is $\mathscr{M}\left\{\left(\mathrm{D}_{0} / \mathrm{Dt}\right) \nabla . \nabla.\right\}$. 
It should be borne in mind that although all these multipoles may appear in the expansion, those to be naturally expected are the volume displacement and the volume velocity monopole and the volume acceleration dipole and quadrupole (this one being the principal source in a number of problems in aeroacoustics). Other terms may appear if one of these distributions has a particular form that degenerates into a higher order source or that, combined with another term, yelds a different order (or type of) source.

An interesting example for the operators $\mathscr{M}$ and $\mathscr{D}$ in a moving medium is given by Lilley's equation [2], where the operator describes the propagation of sound in an undirectional, transversely sheared flow, the mean velocity field $\mathrm{U}$ being given by $\mathbf{U}(\mathbf{x})=\mathbf{U}\left(\mathrm{x}_{2}, \mathrm{x}_{3}\right) \mathbf{e}_{1}$. In this case, $\mathscr{L}$ refers to a third order wave operator and $\mathscr{M}$ and $\mathscr{D}$. are given by

$$
\mathscr{K}=\frac{\mathrm{D}_{0}^{2}}{\mathrm{D} \mathrm{t}^{2}} \quad \mathscr{D} .=-\left(\frac{\mathrm{D}_{0}}{\mathrm{Dt}} \nabla-2 \nabla \mathrm{U} \frac{\partial}{\partial \mathrm{x}_{1}}\right) .
$$

It is evident the proper dipole (or quadrupole) term envolves now two terms, neither of them alone having meaning as a source. Their interdependence has been pointed out by a number of authors.

Another example of a two-term dipole operator is found in Howe's acoustic analogy [3], which refers to the propagation in an irrotacional homoentropic mean flow. The non-linearized $\mathscr{D}$. is

$$
\mathscr{D} .=-\left(\nabla-\frac{1}{\mathrm{c}^{2}} \frac{\mathrm{D} \mathbf{v}}{\mathrm{D} t}\right)
$$

although in this case, the second term is always abandoned for being smaller than the first.

It is not uncommon to find papers in aeroacoustics where source terms that bear resemblance to a (volume acceleration) quadrupole but fail to present the appropriate operator $-\mathscr{D}$.( $\nabla$.) are treated as one. These terms, which alone do not posses a physical meaning, are often found after the neglect of their complementary parts which, on their own, also lack a sound interpretation. A situation where this procedure has led to incorrect results and conclusions has been recently discussed in [4]. Sometimes grouping of terms after further use of the continuity and momentum equations followed by the transference to the left hand side of some of the resulting terms also have the effect of producing non interpretable source terms. The author believes that a good equivalent source term should be amenable to be identified as such at the fundamental equations themselves, the source terms thus found being more than mathematical entities.

\section{3 - The Wave Operator}

Equations (1) and (2) suggest the existence of a close relationship between the structure of the source function and that of the wave operator: as the terms in (1) will depend on a single fluctuating quantity $\pi$, it is quite tempting to expect that $\mathscr{L}$ can be writen as

$$
\mathscr{L}[\pi]=\mathscr{M}\left(\mathrm{g}_{1}[\pi]\right)+\mathscr{D} \cdot\left(\mathrm{g}_{2}[\pi]\right)=\mathrm{Q}
$$

where, if $\pi$ refers basically to density fluctuations, $g_{1}$ stands for a time derivative and $g_{2}$ for $c_{0}{ }^{2} \nabla$; if $\pi$ refers to pressure fluctuations, these operators are to be multiplied, on the left side, by $\mathrm{c}_{0}{ }^{-2}$.

Equation (6) says that an acoustic wave operator (that conforms with it) can be interpreted as being composed of monopole and dipole operators, i.e., that the wave equation can be written as a sum of monopole and dipole source fields, which defines the source Q. Indeed, as the chief candidate for $\mathrm{g}_{1}$ is $\mathrm{D}_{0} / \mathrm{Dt}$ (or $\mathrm{c}_{0}{ }^{-2} \mathrm{D}_{0} / \mathrm{Dt}$ ), the first term is basically a volume displacement monopole, while the second one, if $\pi$ is a pressure-type variable or, otherwise, if $\mathrm{c}_{0}{ }^{2}$ is uniform, degenerates into an isotropic volume acceleration quadrupole, so that the equation can be related to the difference of these source fields. Both these sources are associated with volume expansions, their sound fields being, in an homogeneous medium at rest, equal everywhere but at the origin of the perturbation, that is, at the source. 
Among the formulations for aerodynamically generated sound in a moving medium, some approaches reproduce the structure depicted in (6) while others deviate from it, to a lesser or greater extent, due mainly to the choice of the dependent variable and/or to an "excessive" rearrangement of terms.

A most interesting example is again provided by Lilley's equation. Then, $\pi$ is given by $\ln \left(\rho / \rho_{\infty}\right)$, $\rho_{\infty}$ being a reference value for $\rho, \mathrm{g}_{1}=\mathrm{D}_{0} / \mathrm{Dt}$ and $\mathrm{g}_{2}=\mathrm{c}_{0} 2 \mathrm{~V}$. What is quite surprising is that the non-linear monopole and dipole terms (given by $q=I_{0}-I, f=\Pi_{0}-\Pi$ ) combine in the source function to form another set of exact (and more interesting) monopole, dipole and quadrupole terms [4]. These terms also involve minus the wave operator applied to $\pi^{2} / 2$, what corroborates Goldstein's suggestion [5] of using a modified variable that, to second order, describes the isentropic density fluctuations $p^{\prime} / c^{2}$. This finding also suggests that the equation is written in a kind of optimized form.

In Howe's analogy, on the other hand, the choice of the dependent variable as the specific stagnation enthalpy $\mathrm{B}$ has the consequence that while the natural monopole operator $\mathscr{K}$ is actually $\partial / \partial$ t, the monopole term in $\mathscr{L}$, in its full non-linear form, appears as

$$
\mathscr{M}\left(\mathrm{g}_{\mathrm{I}}[\mathrm{B}]\right)=\frac{\mathrm{D}}{\mathrm{Dt}}\left(\frac{1}{\mathrm{c}^{2}} \frac{\mathrm{DB}}{\mathrm{Dt}}\right)
$$

what is partly due to the fact that, in the isentropic approximation, one has $(1 / \rho) \partial \mathrm{p} / \partial \mathrm{t}=\mathrm{DB} / \mathrm{Dt}$.

It is expected that the comparison of different formulations for the aerodynamical noise problem with equation (6) can be valuable in the investigation of their adequacy and strenght. Although it may be too naive the expect that a good formulation must necessarily comply with it, it seems quite reasonable to think that, at least in some appropriate limit, it must reduce to a very similar form. A careful analysis of the deviations may help in improving the concepts presented herein and also contribute to the achievement of a better insight on the question of defining equivalent sound sources in aeroacoustics.

\section{CONCLUSION}

The derivation of the non-homogeneous acoustic wave equation has been examined, it being pointed out that, for a moving medium, there is a natural way of expressing source terms. As a consequence, the terms resulting from the multipole expansion of a general source term may lack physical meaning. Since any source term in the wave equation can be traced back to the right hand sides of the continuity or momentum equations, representing fundamental sources, these are the appropriate terms to be expanded, each originating a different family of multipoles. The analysis suggests that a good equivalent source term in aeroacoustics should, preferably, be liable to be identified at the fundamental equations themselves (sometimes by working backwards). It also evidentiates that the wave operator has a structure that mirrors that of the source function and which can be related, ideally, to the difference of the source fields of a volume displacement monopole distribution and of an isotropic volume acceleration quadrupole distribution. It is expected that the investigation of the actual structure of the operator in different situations and the comparison with the ideal form presented herein may lead to a better understanding of both source and propagation mechanisms.

Acknowledgment: This investigation was supported by the National Research Council of Brazil, CNPq.

\section{REFERENCES}

[1] Pierce, A. D., Acoustics - An Introduction to its Physical Principles and Aplications, $2^{\text {nd }}$ ed. (A.S.A., N.Y.), 1989.

[2] Lilley, G. M., On the Noise from Jets, AGARD Conf. Proc. on Noise Mechanisms (Bruxelles, 1973), paper 13, 1974.

[3] Howe, M. S., J. Fluid Mech., 71(4), pp. 625-673, 1975.

4] Musafir, R. E., A Note on the Description of Jet Noise Source Terms, Proc. Inst. of Acoust., $15(3)$, pp. 901-908, 1993.

[5] Goldstein, M. E., Aeroacoustics of Turbulent Shear Flows, Ann. Rev. Fluid Mech., 16, pp. $263-285,1984$. 